\title{
In vivo biodistribution, anti-inflammatory, and hepatoprotective effects of liver targeting dexamethasone acetate loaded nanostructured lipid carrier system
}

\author{
This article was published in the following Dove Press journal: \\ International Journal of Nanomedicine \\ 26 July 2010 \\ Number of times this article has been viewed
}

\begin{abstract}
Min-ting Wang'
Yun Jin'

Yun-xia Yang ${ }^{2}$

Chun-yan Zhao'

Hong-yun Yang'

Xue-fan Xu'

Xuan Qin'

Zhao-dan Wang ${ }^{2}$

Zhi-rong Zhang'

Yan-lin Jian ${ }^{3}$

Yuan Huang'

'Key Laboratory of Drug Targeting and Drug Delivery System, Ministry of Education, West China School of Pharmacy, ${ }^{2}$ West China School of Preclinical and Forensic Medicine, Sichuan University, Chengdu, People's Republic of China; ${ }^{3}$ Shenzhen Second People's Hospital, Shenzhen, People's Republic of China
\end{abstract}

Correspondence: Yuan Huang Key Laboratory of Drug Targeting and Drug Delivery System, Ministry of Education, West China School of Pharmacy, Sichuan University, No. 17, Block 3, Southern Renmin Road, Chengdu 6I004I, P.R. China

Tel +86-28-8550I617

Fax +86-28-85501617

Email huangyuan0@yahoo.com.cn

Yan-lin Jian

Shenzhen Second People's Hospital

No. 3002, Sungangxi Road Shenzhen

5 I803I, P.R. China

Tel +86-28-83225I 66

Fax +86-28-83225I66

Email gdszjyl@।63.com

\begin{abstract}
We aimed to evaluate whether the enhancement of the liver accumulation and anti-inflammatory activity of dexamethasone acetate (DXMA) could be achieved by incorporating it into nanostructured lipid carrier (NLCs). DXMA-NLCs were prepared using a film dispersion-ultrasonication method and characterized in terms of particle size, PDI, zeta potential, differential scanning calorimetry, drug loading capacity, encapsulation efficiency, and in vitro release. The biodistribution and pharmacokinetics of DXMA-NLCs in mice were significantly different from those of the DXMA solution (DXMA-sol). The peak concentration of DXMA-NLCs was obtained half an hour after intravenous administration. More than $55.62 \%$ of the total administrated dose was present in the liver. An increase of 2.57 fold in the area under the curve was achieved when compared with that of DXMA-sol. DXMA-NLCs exhibited a significant anti-inflammatory and hepatoprotective effect on carrageenan-induced rats and carbon tetrachloride-induced mice compared with DXMA-sol. However, the effect was not in proportion to the dosage. The intermediate and low dosages presented better effects than DXMA-sol. All results indicate that NLCs, as a novel carrier for DXMA, has potential for the treatment of liver diseases, increasing the cure efficiency and decreasing the side effects on other tissues.
\end{abstract}

Keywords: dexamethasone acetate, nanostructured lipid carrier, liver targeting

Inflammatory processes are involved in a large number of liver diseases and may lead to liver fibrosis and cirrhosis. ${ }^{1}$ Dexamethasone acetate (DXMA) is a synthesized glucocorticoid, which has been used as an anti-inflammatory drug in the treatment of chronic active hepatitis for the prevention of liver fibrosis. ${ }^{2}$ Although DXMA enhances hepatocyte viability and improves the expression of liver-specific genes in liver diseases, the response rates obtained from a series of recent clinical trials were poor, which can be explained by the following factors. Firstly, DXMA has poor water solubility and is almost insoluble in most physiologically compatible and pharmaceutically acceptable solvents, both of which limit its clinic applications. ${ }^{3}$ Secondly, DXMA does not act specifically on liver cells, and long term use of large doses may lead to many side effects, such as osteoporosis, an increased risk of infections, and Cushing's syndrome, especially during chronic use. ${ }^{1}$ To overcome these problems, micro/ nanoparticulate delivery systems were utilized for the encapsulation of DXMA. Polytrimethylene carbonate and PLGA were used to improve the aqueous solubility and in vitro release of dexamethasone, but in vivo results were not reported. ${ }^{46}$ 
Particulate carriers usually accumulate in the liver by passive targeting upon parenteral administration. ${ }^{7}$ Oxymatrine and 5-fluorouracil prodrug were incorporated in solid lipid nanoparticles (SLNs) and showed higher liver targeting efficacy compared with free drug. ${ }^{89}$ Nanostructured lipid carriers (NLCs) are the second generation of lipid nanoparticles, composed of a binary mixture of solid lipid and a spatially different liquid lipid as a carrier. ${ }^{6}$ Though the major advantages of NLCs include increased drug loading capacity and decreased drug expulsion compared with SLNs, to date, very few researchers have systematically explored the in vivo liver targeting of NLCs.

Recently, we developed liver targeting DXMA-NLCs by a film dispersion-ultrasonication method. In this study we aimed to evaluate whether the enhancement of the liver accumulation and anti-inflammatory activity of DXMA could be obtained by incorporated into NLCs. The biodistribution and pharmacokinetics of DXMA-NLCs were studied in normal mice and the therapeutic efficacy was investigated on carrageenan-induced rats and carbon tetrachloride-induced mice for the first time.

\section{Materials and methods Chemicals and animals}

DXMA was supplied by Tianyao Pharmaceutical Co. Ltd. (Tianjin, P.R.C.). Pluronic 188 (F68) and carrageenan were purchased from Sigma (St. Louis, MO, USA). Purified egg yolk lecithin (Lipoid E80) was obtained from LIPOID GMBH (Ludwigshafen, Germany). Soybean oil for parenteral use was supplied by Beiya Co. Ltd. (Tieling, P.R.C.) and tween 80 from Bodi Chemicals Co. Ltd. (Tianjin, P.R.C.). Carbon tetrachloride $\left(\mathrm{CCl}_{4}\right)$ was obtained from Kelong Chemicals Co. Ltd. (Chengdu, P.R.C.). Alanine aminotransferase, aspartate aminotransferase, and alkaline phosphatase assay kits were purchased from Jiancheng Institute of Biotechnology (Nanjing, P.R.C.). A hydroxyproline assay kit was supplied by Bei Fang Wei Ye Co. Ltd. (Beijing, P.R.C.). All other chemicals were of analytical grade.

Kunming mice weighing between 18 and $22 \mathrm{~g}$ and Wistar rats weighing between 180 and $200 \mathrm{~g}$ were purchased from the Laboratory Animal Center of Sichuan University (Chengdu, P.R.C.).

\section{Preparation of DXMA loaded NLCs}

DXMA-NLCs were prepared by a film dispersionultrasonication method. ${ }^{10}$ Briefly, $15 \mathrm{mg}$ of DXMA, $100 \mathrm{mg}$ of Soybean oil (SO), and $100 \mathrm{mg}$ of lecithin were dissolved in $\mathrm{CH}_{2} \mathrm{Cl}_{2}$. The solution was evaporated under reduced pressure to remove the solvent and form a thin layer of uniform film on the wall of a round-bottom flask. A total of $20 \mathrm{~mL}$ of aqueous phase containing surfactants $\mathrm{F} 68(2 \%, \mathrm{w} / \mathrm{v})$ and tween $80(2 \%, \mathrm{w} / \mathrm{v})$ was added to disperse the film. The mixture was then further dispersed ultrasonically for 5 minutes to obtain NLCs. DXMA-containing NLCs were then purified by filtration through a cellulose acetate membrane $(0.8 \mu \mathrm{m})$ to eliminate drug crystals. After purification, the NLC dispersions were freeze-dried for storage with mannitose as the cryoprotector.

\section{Characterization of NLCs}

The NLCs were characterized for their size, PDI, and zeta potential with a Malvern Zatasize NanoZS90 (Malvern Instruments Ltd., Malvern, UK). Differential scanning calorimetry (DSC) and ${ }^{31} \mathrm{P}$ Nuclear Magnetic Resonance Spectroscopy ( ${ }^{31} \mathrm{P}$ NMR) of blank NLCs, DXMA, DXMA-NLCs, and a physical mixture of blank NLCs and DXMA (the same ratio as that of the DXMA-NLCs) were performed on a DSC 204 (NETZSCH, Selb, Germany) and an AV300 spectrometer (Bruker, Rheinstetten, Germany).

The drug loading capacity (LC\%) and encapsulation efficiency (EE\%) were determined in triplicate by HPLC. The freeze-dried samples were redispersed in a solution of methanol/water (7:3, v/v) and sonicated. Subsequently, the suspensions were centrifuged $(11,100 \times \mathrm{g}, 5$ minutes $)$, and the supernatant was assayed by HPLC for the total amount of DXMA in lipid nanoparticles. The amount of DXMA contained in the NLCs was determined by resuspending the same freeze-dried samples in $1 \mathrm{~mL}$ water, adding $0.2 \mathrm{~mL}$ $\mathrm{NaCl}$ solution $(0.9 \%)$ to salt out the unincorporated drugs and centrifuging $(18,000 \times \mathrm{g}, 20$ minutes). The upper suspensions were then added to methanol, sonicated, and centrifuged $(11,100 \times \mathrm{g}, 5$ minutes). Finally, the supernatant was tested by the same HPLC method for the determination of DXMA entrapped within the NLCs. The HPLC system consisted of an Alltech Model 426 HPLC Pump, an Alltech UVIS-201 Absorbance Detector, and an Allchrom plus Client/Server data operator (Alltech International, Inc., Virginia, USA). The mobile phase contained a ratio of methanol: water of 70:30. A Shimpack CLC-ODS C18 column $(150 \times 4.6 \mathrm{~mm}$, $5 \mu \mathrm{m}$, Dikma Diamonsil ${ }^{\circledR}$, Dikma Inc., Shanghai, P.R.C) was eluted at a flow rate of $1.0 \mathrm{~mL} / \mathrm{min}$. The eluate was monitored at $30^{\circ} \mathrm{C}$ and DXMA was assayed at a wavelength of $240 \mathrm{~nm}$. $\mathrm{EE} \%$ and LC\% of DXMA loaded NLCs were calculated as given below:

$\mathrm{EE} \%$ = amount of DXMA encapsulated in NLC/total amount of DXMA in lipid nanoparticles $\times 100 \%$. 
LC $\%$ amount of DXMA encapsulated in NLC/total amount of NLC $\times 100 \%$.

\section{In vitro release study of DXMA-NLCs}

The Visking bag method was used to investigate the in vitro release of DXMA loaded NLCs. Briefly, DXMA-NLCs were resuspended in $5 \mathrm{~mL}$ water at a concentration of $100 \mu \mathrm{g} / \mathrm{mL}$, transferred into the dialysis bag and dialyzed against $45 \mathrm{~mL}$ phosphate buffer ( $\mathrm{pH} 7.4$, containing $0.03 \mathrm{wt} \%$ SDS). During dialysis, a constant temperature shaker (Shenzhen Worldwide Industry, Co. Ltd., Shenzhen, P.R.C) was used, thermostatted at $37 \pm 1{ }^{\circ} \mathrm{C}$ and mechanically shaken at a suitable rotation speed.

At designated time intervals, $1 \mathrm{~mL}$ samples of the dialysis medium was taken for HPLC measurement of DXMA and the same volume of fresh medium was added. The release experiments were performed in triplicate.

\section{In vivo biodistribution and pharmacokinetic studies of DXMA-NLCs}

The Sichuan University animal ethical experimentation committee approved all procedures of the in vivo studies, according to the requirements of the National Act on the use of experimental animals (P.R.C.). Kunming mice received a single intravenous (iv) injection of DXMA-NLCs or DXMA solution at a DXMA equivalent dose of $10 \mathrm{mg} / \mathrm{kg}$. The mice were sacrificed at $0.08,0.25,0.5,1,2,4,6,8,10$, and 12 hours after iv administration. Blood samples were collected from the orbit venous plexus and plasma samples were obtained by centrifugation at $5,000 \times \mathrm{g}$ for 10 minutes. The tissues, including heart, lung, liver, spleen, and kidney were harvested and accurately weighed and homogenized. Plasma and tissue samples within the same group were pooled together for further analysis $(\mathrm{n}=5)$.

The DXMA concentrations in the plasma or tissues were determined by an HPLC assay. The extraction procedure was as follows. Plasma or tissue homogenates $(0.5 \mathrm{~mL})$ were mixed with $0.1 \mathrm{~mL}$ methyltestosterone solution $(8.66 \mu \mathrm{g} / \mathrm{mL})$ as the internal standard and $0.2 \mathrm{~mL}$ phosphate buffer $(\mathrm{pH}=2.0)$. The mixture was vortexed with $3 \mathrm{~mL}$ acetoacetate and then centrifuged $(5,900 \times \mathrm{g}, 10$ minutes). A total of $2 \mathrm{~mL}$ of supernatant was evaporated to dryness. Finally, the residue was redissolved in $0.2 \mathrm{~mL}$ methanol, following centrifugation at $14,000 \times \mathrm{g}$ for 10 minutes. Following this, $20 \mu \mathrm{L}$ of the clear supernatant was injected into the HPLC system as described above.

Plasma and tissue concentration data obtained were pooled to provide mean concentration data. Pharmacokinetic parameters in plasma and organs were calculated using a statistical moment algorithm and were obtained by calculating the area under the concentration-time curve (AUC) using the linear trapezoidal rule and extrapolating this value to infinity by dividing the last measurable concentration by the elimination rate constant.

\section{The anti-inflammatory effect of DXMA-NLCs on carrageenan-induced rats}

An anti-inflammatory test of DXMA-NLCs was performed according to the method developed by Lange et al. ${ }^{11}$ Wistar rats were randomly divided into 5 groups $(\mathrm{n}=8)$. Group 1 (control) received saline; groups 2 and 3 were injected with DXMA-NLCs (DXMA equivalent dose of $3 \mathrm{mg} / \mathrm{kg}$ or $0.6 \mathrm{mg} /$ $\mathrm{kg}$ ); and groups 4 and 5 received DXMA-sol $(3 \mathrm{mg} / \mathrm{kg}$ or $0.6 \mathrm{mg} / \mathrm{kg}$ ). Drugs and saline were given through the tail veins 1 hour before subcutaneous injection of carrageenan into the right hind paws. The paw volume was measured before drug administration and then at $0.5,1,2,3,4,5$, and 6 hours after carrageenan injection, using a digital plethysmometer. The amount of paw swelling was determined from time to time and expressed as follows:

Inhibition ratio $\%=$ (edema value of control group - edema value of drug group)/edema value of control group $\times 100 \%$.

\section{Hepatoprotective effects of DXMA-NLCs on carbon tetrachloride-induced mice}

\section{Animal treatments}

Kunming mice were divided into 8 groups. Group 1 received normal saline and served as the control. Animals in groups 2, $3,4,5,6$, and 7 were subcutaneously injected with carbon tetrachloride $\left(40 \% \mathrm{CCl}_{4}\right.$ in olive oil, $\left.0.2 \mathrm{~mL} / 10 \mathrm{~g}\right)$ on the first day and then $0.1 \mathrm{~mL} / 10 \mathrm{~g}$ once every 5 days for a period of 4 weeks. Group 2 received normal saline and served as a $\mathrm{CCl}_{4}$ intoxication control ( $\mathrm{CCl}_{4}$ group). Group 3 received blank NLC and served as the vehicle control. Groups 4 and 5 received DXMAsol at doses of $2 \mathrm{mg} / \mathrm{kg}$ and $1 \mathrm{mg} / \mathrm{kg}$, respectively. Groups 6 , 7, and 8 received DXMA-NLCs at a DXMA equivalent dose of $2 \mathrm{mg} / \mathrm{kg}, 1 \mathrm{mg} / \mathrm{kg}$, and $0.5 \mathrm{mg} / \mathrm{kg}$, respectively. The normal saline and drugs were administered through the tail veins once per week over a period of 4 weeks from the second day after injection of carbon tetrachloride.

Blood samples were collected from the orbit venous plexus 24 hours after the last treatment of drugs and were used for biochemical evaluations. The mice were 
then dissected and the livers were removed for the calculation of liver coefficients and then homogenized for the determination of hydroxyproline content.

\section{Biochemical evaluations}

Liver damage was assessed by estimating serum activities of alanine aminotransferase (ALT), aspartate aminotransferase (AST), and alkaline phosphatase (ALP) using commercially available test kits. The results were expressed as activity (IU/100 mL).

\section{Liver coefficients}

After weighing the body and liver, the ratio of wet liver weight ( $\mathrm{mg}$ ) to total body weight ( $\mathrm{g}$ ) (liver coefficient) was calculated.

\section{Hydroxyproline content}

Hydroxyproline (Hyp) content in liver was determined by the Hyp assay kit spectrophotometric method according to the manufacturer's instructions. Data was expressed as Hyp ( $\mu \mathrm{g}) /$ wet liver weight $(\mathrm{g})$.

\section{Statistical analysis}

Data were expressed as mean and standard deviation (SD) and statistically assessed using a one-way analysis of variance (ANOVA). Differences between the $\mathrm{CCl}_{4}$ group and the drug or NLC-treated groups were evaluated by the Student's $t$ test, with a significance level of $P<0.05$.

\section{Results and discussion Preparation and characterization of DXMA-NLCs}

DXMA-NLCs were successfully prepared by the film dispersion-ultrasonication method using soybean oil and egg yolk lecithin as lipid materials with a mean particle size of $151 \pm 3.5 \mathrm{~nm}$, a PDI of $0.215 \pm 0.083$, a zeta potential of $-38.7 \pm 3.7 \mathrm{mv}$, an encapsulation efficiency of $97.11 \pm 0.51 \%$, and a drug loading capacity of $4.41 \pm 0.04 \%$. The in vitro release study revealed sustained release of drugs from DXMA-NLCs in contrast with the free DXMA (Figure 1). While about $100 \%$ of the free drug was found in release medium after approximately 2 hours, less than $10 \%$ of the drug released at 1 hour and about $50 \%$ of the drug released within 12 hours from DXMA-NLCs.

DSC analysis was performed to evaluate possible interactions between DXMA and the lipid materials (Figure 2). Blank NLCs exhibited 3 melting points at $47^{\circ} \mathrm{C}$, $157.2^{\circ} \mathrm{C}$, and $167^{\circ} \mathrm{C}$ (Figure 2A). Similarly, drug loaded
NLCs presented melting points at $46.1^{\circ} \mathrm{C}, 157.5^{\circ} \mathrm{C}$, and $167.1^{\circ} \mathrm{C}$ (Figure 2B). In addition, the typical transitions of physical mixture were $49.1^{\circ} \mathrm{C}, 151.5^{\circ} \mathrm{C}$, and $166.7^{\circ} \mathrm{C}$ (Figure 2C). DXMA alone showed a peak at $233.3^{\circ} \mathrm{C}$ as seen in Fig. 2D. No considerable difference could be observed among blank NLCs, drug-loaded NLCs, and physical mixture, which means the loading of a small amount of drug on NLCs did not change the melting points significantly. The DSC thermograms of physical mixture and the drug-loaded NLCs did not show a melting peak for DXMA. This absence may be induced by the low amount of the drug $(4.41 \pm 0.04 \%)$ in DXMA-NLCs, which was coincident with the research of $\mathrm{Mu}$ et al. ${ }^{12}$ Their team reported difficulties in detecting the endothermic peak of drug in microspheres when the drug ratio is lower than $10 \%$.

To further pursue the interaction between the drug and lipids, ${ }^{31} \mathrm{P}$ NMR spectral phospholipid profiles of blank NLCs, DXMA, DXMA-NLCs, and physical mixtures of blank NLCs and DXMA were investigated (Figure 3). The spectrum obtained from DXMA indicated there was no phosphonium in DXMA. A resonance around $0.40 \mathrm{ppm}$ was easily observed in all the spectrum of blank NLCs, DXMA-NLCs, and the physical mixture, which was obtained from the same $P$-enriched fraction. This resonance indicated there is no significant chemical environmental difference of $P$ among blank NLCs, DXMA-NLCs, and the physical mixture. Hence, there was no chemical interaction between phospholipid and other materials including the drug.

\section{Biodistribution and pharmacokinetics in mice}

The biodistribution and pharmacokinetics of DXMA-NLCs were examined in mice. The percentage of the total administered dose (TAD) of free DXMA and NLCs in plasma and tissues at different times after iv

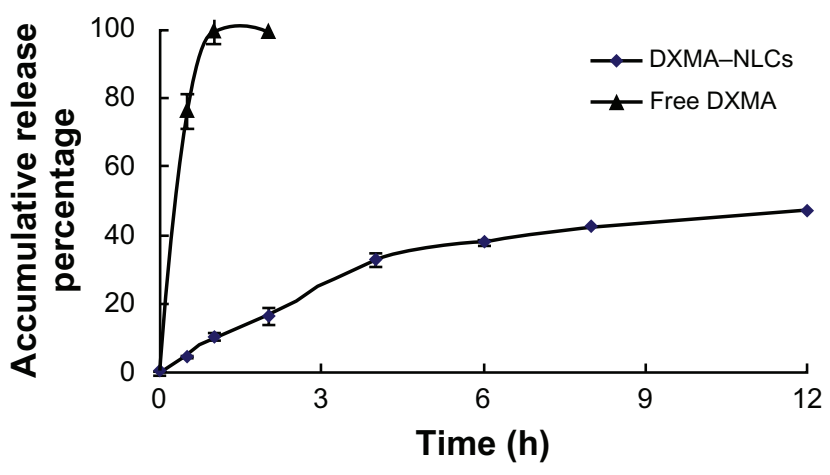

Figure I In vitro release profiles of DXMA from DXMA-NLCs and free DXMA (mean $\pm S D, n=3$ ). 

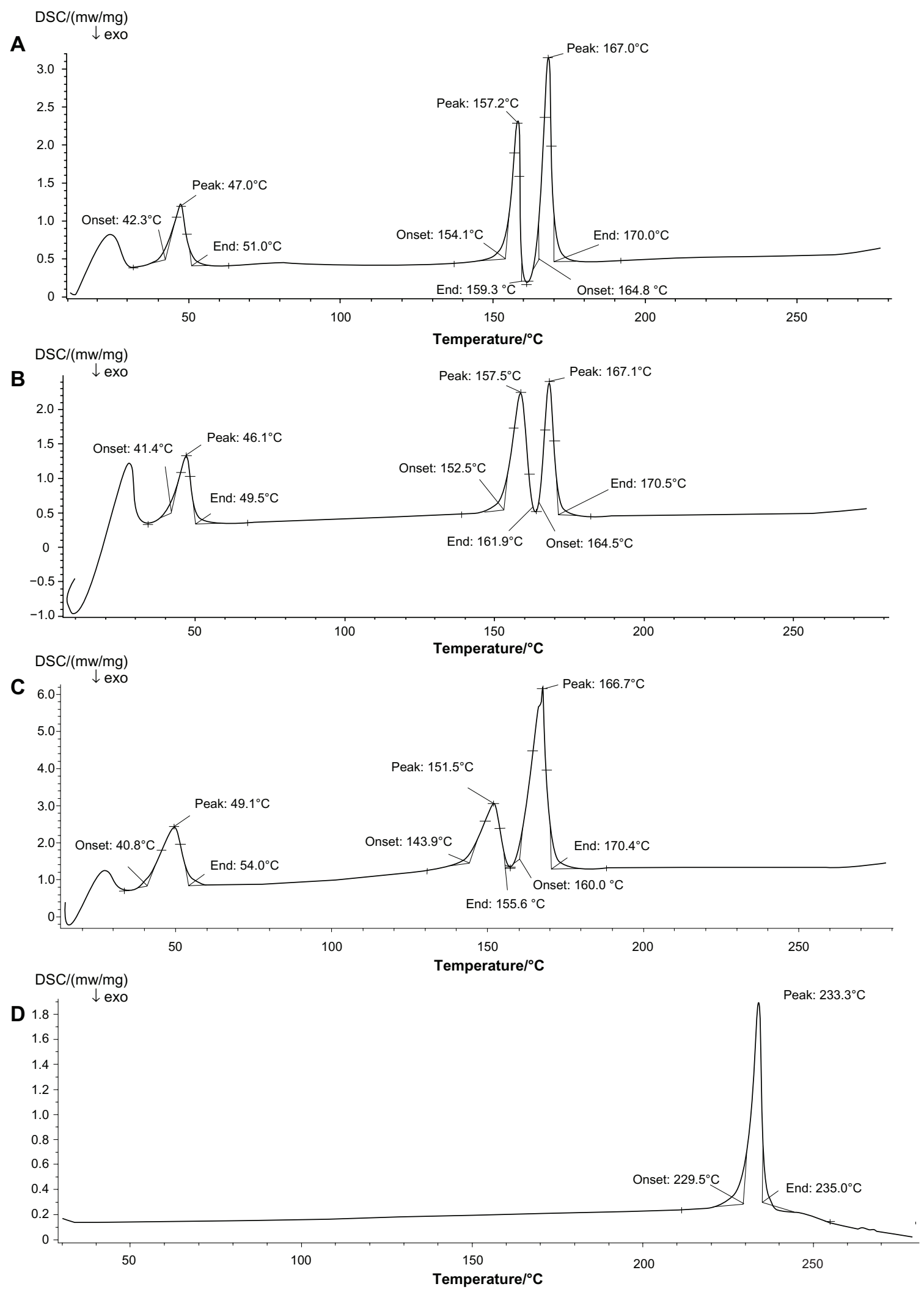

Figure 2 Differential scanning calorimetry (DSC) of Blank NLCs (A), DXMA-NLCs (B), physical mixture of blank NLCs and DXMA (C), and DXMA (D). 
DXMA

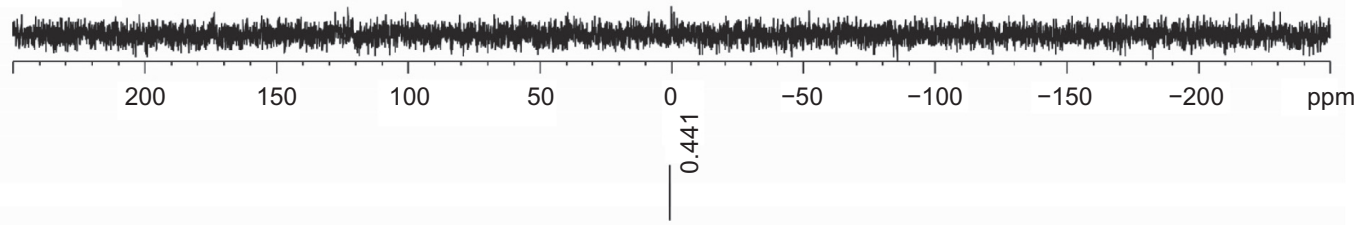

BLANK-NLC
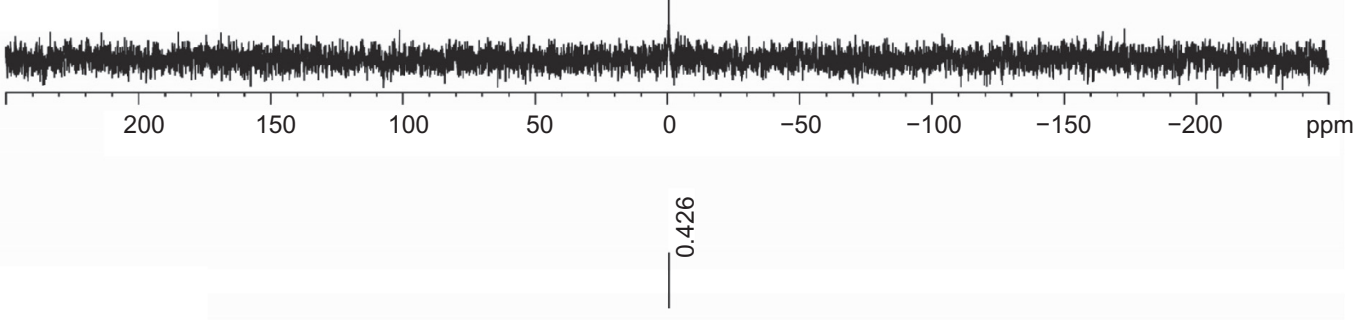

DXMA-NLC
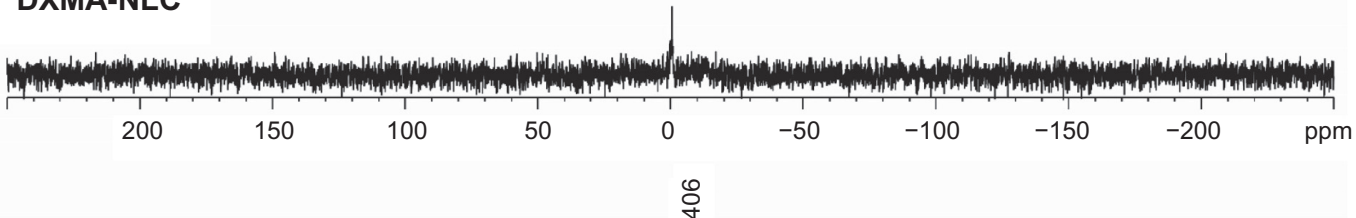

BLANK-NLC+DXMA

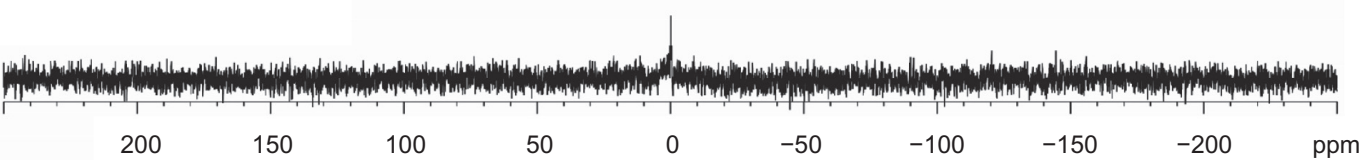

Figure 3 The ${ }^{31}$ P NMR spectrums of DXMA, blank NLCs, DXMA-NLCs, and physical mixture of blank NLCs and DXMA.

administration are summarized in Table 1. The DXMA levels after intravenous injection of DXMA-NLCs or DXMA solution in various organs are illustrated in Figure 4. Different compartment models were used to describe the pharmacokinetics of DXMA and DXMANLCs in plasma and tissues. The calculated data were closest to observed data when a 2-compartment model was applied. The main pharmacokinetic parameters in plasma and tissues are shown in Table 2.

The results shown in Figure 4A clearly indicate that DXMA-NLCs were preferentially accumulated in the liver. The peak concentration of DXMA-NLCs was reached at 0.5

Table I Percentage of TAD of DXMA in plasma and tissues after iv administration of DXMA-NLCs (A) and DXMA-sol (B) in mice

\begin{tabular}{|c|c|c|c|c|c|c|c|c|c|c|}
\hline Time (hours) & 0.08 & 0.25 & 0.5 & I & 2 & 4 & 6 & 8 & 10 & 12 \\
\hline Heart A & 9.89 & 12.54 & 15.67 & 5.64 & 4.52 & 3.06 & 0.77 & 0.71 & 0.00 & 0.00 \\
\hline Heart B & 14.46 & 9.29 & 6.63 & 3.39 & 1.41 & 1.18 & 0.00 & 0.00 & 0.00 & 0.00 \\
\hline Liver A & 39.10 & 47.61 & 55.62 & 27.55 & 17.18 & 7.31 & 3.31 & 2.84 & 4.31 & 2.21 \\
\hline Liver B & 37.35 & 35.43 & 34.72 & 17.91 & 10.02 & 2.19 & 0.00 & 0.00 & 0.00 & 0.00 \\
\hline Spleen A & 7.94 & 10.83 & 10.43 & 5.36 & 4.57 & 0.49 & 0.44 & 0.00 & 0.00 & 0.00 \\
\hline Spleen B & 9.86 & 7.99 & 5.62 & 4.93 & 3.38 & 0.51 & 0.00 & 0.00 & 0.00 & 0.00 \\
\hline Lung A & 9.96 & 12.42 & 9.12 & 4.29 & 3.29 & 0.74 & 0.00 & 0.00 & 0.00 & 0.00 \\
\hline Lung B & 10.21 & 8.68 & 9.26 & 5.46 & 2.41 & 1.38 & 0.00 & 0.00 & 0.00 & 0.00 \\
\hline Kidney A & 10.81 & 5.51 & 6.29 & 1.78 & 1.01 & 0.91 & 0.39 & 0.00 & 0.00 & 0.00 \\
\hline Kidney B & 11.54 & 9.70 & 7.10 & 1.59 & 2.00 & 0.54 & 0.00 & 0.00 & 0.00 & 0.00 \\
\hline Plasma A & 24.19 & $17.6 \mid$ & 13.99 & 14.88 & 7.87 & 1.85 & 1.14 & 0.00 & 0.00 & 0.00 \\
\hline Plasma B & 17.79 & 18.11 & 11.58 & 10.93 & 8.38 & 1.40 & 0.00 & 0.00 & 0.00 & 0.00 \\
\hline Brain A & 3.41 & 2.38 & 2.38 & 0.36 & 0.00 & 0.00 & 0.00 & 0.00 & 0.00 & 0.00 \\
\hline Brain B & 2.77 & 3.01 & 2.88 & 0.72 & 0.00 & 0.00 & 0.00 & 0.00 & 0.00 & 0.00 \\
\hline
\end{tabular}



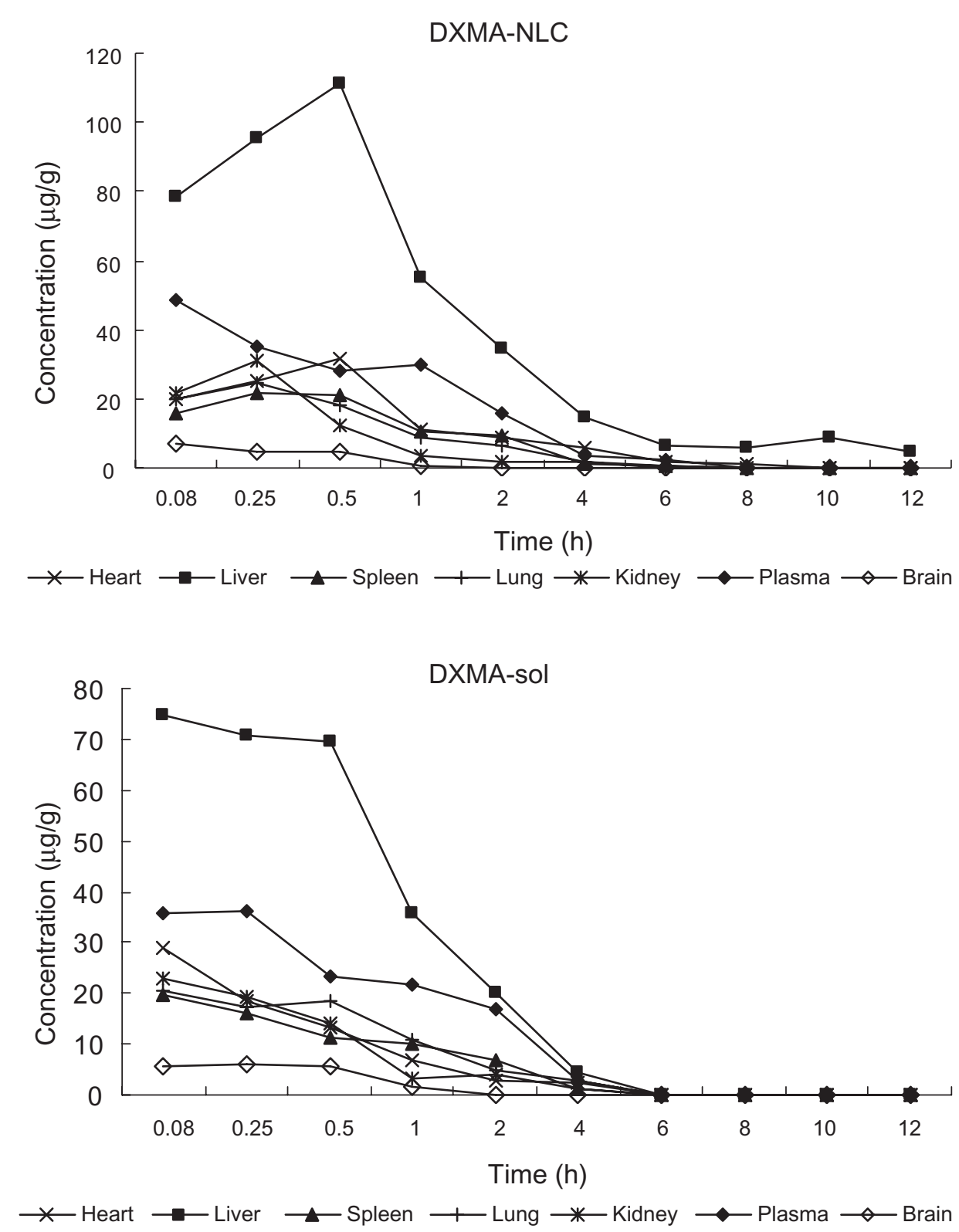

Figure 4 DXMA concentrations in plasma and tissues at different time points after i.v. administration of DXMA-NLCs and DXMA-sol.

hours after iv administration and more than $55.62 \%$ of TAD $\%$ was present in the liver, as shown in Table 1 . In contrast, the peak concentration of free DXMA in the liver occurred at 0.08 hours as illustrated in Table 1 and Figure 4B and only 2.19\% of $\mathrm{TAD} \%$ remained after 4 hours. A 2.57-fold larger area under the curve was achieved for DXMA-NLCs compared with that of DXMA (Table 2). Meanwhile, DXMA-NLCs had an increased $t_{1 / 2}(1.15$ hours $)$ and decreased CL $(0.07$ $\mathrm{L} / \mathrm{h} / \mathrm{kg}$ ) compared to free DXMA ( 0.38 hours, $0.17 \mathrm{~L} / \mathrm{h} / \mathrm{kg}$ ), indicating that NLCs were better retained in the liver than free drug. These results clearly indicate a remarkably selective liver targeting property of DXMA-NLCs, presumably because of the general clearance of nanoparticles by the phagocytic cells. ${ }^{13}$ The RES plays a major role in clearing small foreign particles from blood, which serves for the nanoparticles being passively targeted to certain phagocytic cells. ${ }^{13}$ After intravenous administration with NLCs, they were mostly recognized by macrophages (MPS) owing to the physicochemical characteristics of particle size, surface charge. and surface hydrophobicity. ${ }^{14,15}$ An earlier report by Reddy indicated that negatively charged SLNs have a significantly higher uptake in RES organs, such as the liver and spleen. ${ }^{16}$ Our results for NLCs were in agreement with that report. At the same time, because the SLN uptake by RES is mediated by particle adsorption of blood proteins and opsonins, ${ }^{17}$ increasing the hydrophobicity of the particles 
Table 2 The pharmacokinetic parameters of DXMA-NLCs (A) and DXMA-sol (B) in tissues and plasma

\begin{tabular}{|c|c|c|c|c|c|c|}
\hline \multirow[t]{2}{*}{ Tissues } & \multicolumn{2}{|c|}{ AUC ( $\left.\mu g^{*} h / g\right)$} & \multicolumn{2}{|c|}{$T_{1 / 2}(h)$} & \multicolumn{2}{|c|}{ CL (L/h/kg) } \\
\hline & $\mathbf{A}$ & B & A & B & $\mathbf{A}$ & B \\
\hline Heart & 29.84 & 13.91 & $0.7 \mathrm{I}$ & 0.44 & 0.34 & 0.72 \\
\hline Liver & 152.95 & 59.48 & 1.15 & 0.38 & 0.07 & 0.17 \\
\hline Spleen & 13.44 & 15.26 & 0.91 & 0.45 & 0.71 & 0.66 \\
\hline Lung & 16.91 & 17.44 & 69.31 & 0.43 & 0.59 & 0.57 \\
\hline Kidney & 12.42 & 11.93 & 0.64 & 0.35 & 0.81 & 0.84 \\
\hline Plasma & 41.95 & 34.78 & 0.55 & 0.41 & 0.24 & 0.29 \\
\hline Brain & 2.23 & 2.72 & 0.16 & 0.16 & 4.48 & 3.67 \\
\hline
\end{tabular}

should increase the incidence of adsorption. ${ }^{18,19}$ Therefore, in our study, the lipid matrix, with soybean as the core material and lecithin as the surface material, provided hydrophobic surfaces of NLCs, leading to enhanced phagocytosis of NLCs. ${ }^{20}$ These results are similar to those observed with hydrophobic polystyrene particles, which were intensively taken up by cells with phagocytic activity. ${ }^{21-23}$ Similar liver targeting of 9-nitrocamptothecin NLCs has also been demonstrated by Li et al. ${ }^{24}$

In most reported studies, non stealth SLN formulations lead to significantly higher levels of drug remaining in the systemic circulation for longer periods of time compared to the free drug and this was also the case in our study. ${ }^{7}$ The AUC of DXMA delivered by NLCs in plasma was 1.21 -fold higher than in corresponding free drug solutions. The half life in plasma of DXMA was also increased when administered via NLCs.
Although a study of the anti-Parkinsonian bromocriptine NLCs indirectly demonstrated that higher brain levels of bromocriptine were achieved after administration of $\mathrm{NLCs}^{25} \mathrm{a}$ higher drug level in the brain was not observed in our study. The AUC of NLCs $(2.23 \mu \mathrm{gh} / \mathrm{g})$ was lower than that of the free drug $(2.72 \mu \mathrm{gh} / \mathrm{g})$.

\section{The anti-inflammatory effect of DXMA-NLCs on carrageenan- induced rats}

The anti-inflammatory effect of DXMA-NLCs was investigated on carrageenan-induced rats. Figure 5 shows the edema value of DXMA-NLCs and DXMA-sol groups, induced by carrageenan, demonstrating that both DXMA-NLCs and DXMA-sol were able to prevent edema formation compared with the control group $(P<0.05)$. Furthermore, the DXMA-NLCs group exhibited more significant responses at lower-dose from 3 to 6 hours after carrageenan injection than the DXMA-sol group at the same dose $(P<0.05)$. The percent inhibition value 5 hours after administration was found to be significantly higher for DXMA-NLCs $(44.9 \% \pm 1.70 \%)$ compared with DXMA-sol $(14.8 \% \pm 5.5 \%, P<0.05)$. Also, in a study of the anti-inflammatory activity of DXMA incorporated into a lipid emulsion, the effect of a DXMA emulsion at a low dosage was found to be as potent as DXMA sodium phosphate at a high dosage. ${ }^{26}$

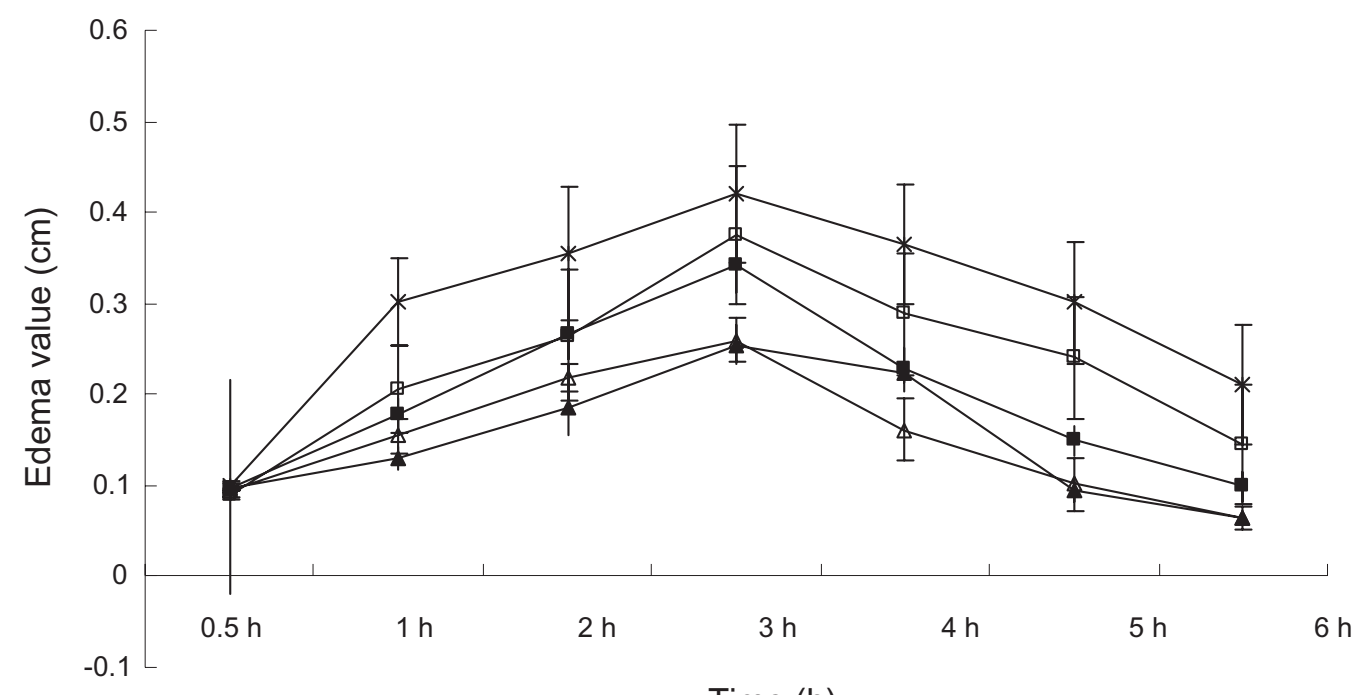

Time (h)

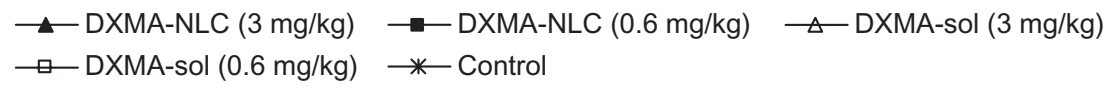

Figure 5 The edema value $(\mathrm{cm})$ of DXMA-NLCs and DXMA-sol on carrageenan induced paw edema in rats. 


\section{Hepatoprotective effects of DXMA-NLCs on carbon tetrachloride-induced mice}

Carbon tetrachloride $\left(\mathrm{CCl}_{4}\right)$ is a classical hepatotoxicant that causes rapid liver damage progressing from steatosis to centrilobular necrosis. Long term administration of $\mathrm{CCl}_{4}$ causes chronic liver injury, and the $\mathrm{CCl}_{4}$-induced animal model is a widely accepted as producing hepatic fibrosis. ${ }^{27,28}$ As shown in Tables 3 and 4, the liver coefficient, contents of Hyp, serum levels of AKP, AST, ALT were all significantly increased after $\mathrm{CCl}_{4}$ treatment, which together imply the successful establishment of the model. The contents of Hyp, serum levels of enzymes in the blank NLC group were close to that of the $\mathrm{CCl}_{4}$ group, suggesting that blank NLC had no obvious impairing effect on the liver. When administered with DXMA and DXMA-NLCs, the aforementioned parameters were all significantly decreased $(P<0.05)$. It should be stressed that the levels of the liver coefficient and Нyp contents of DXMA-NLCs group at the intermediate dose $(1 \mathrm{mg} / \mathrm{kg}$ ) were $56.80 \mathrm{mg} / \mathrm{g}, 0.22 \pm 0.39 \mu \mathrm{g} / \mathrm{mg}$ liver, respectively, which were much lower than those of the DXMA-sol group $(65.25 \mathrm{mg} / \mathrm{g}, 0.66 \pm 0.42 \mu \mathrm{g} / \mathrm{mg}$ liver, $P<0.05)$ with the same dose. Even if no remarkable differences were found for high dose groups between DXMA and DXMA-NLCs, the protecting effect of DXMA-NLCs at intermediate and low dosages was equal to the effect generated by drug solutions at high dosage $(2 \mathrm{mg} / \mathrm{kg}, P>0.05)$. All of these results may be related to the liver accumulation of NLCs. From the aforementioned in vivo biodistribution results, we can see a 2.57-fold larger area under the curve, an increased $t_{1 / 2}$ (1.15 hours), and decreased CL $(0.07 \mathrm{~L} / \mathrm{h} / \mathrm{kg})$ were achieved by DXMA-NLCs compared with that of DXMA (Table 2). Furthermore, contributions from diverse accumulation in the liver may be responsible for the different hepatic protection effect between DXMA-NLCs and DXMA-sol. In this study, DXMA-NLCs with a mean particle size of $151 \pm 3.5 \mathrm{~nm}$, a zeta potential of $-38.7 \pm 3.7 \mathrm{mv}$, and surface hydrophobicity may be mainly taken up by reticuloendothelial systems and transported into nonparenchymal hepatic cells in liver. Kuffer cells, stellate cells, endothelial cells are the members of nonparenchymal hepatic cells, which play a pivotal role in continuing the inflammation with releasing cytokine. Interference with this cytokine release by DXMA could inhibit cells factor release and relive hepatic inflammation. However, for free DXMA, Melgert et al reported it is always accumulated in parenchymal cells which are not the main producers of collagens. ${ }^{1}$ Although further experiments concerning the hepotoprotecting effect mechanism of DXMA-NLCs are
Table 3 Liver coefficient and hydroxyproline (Hyp) content of $\mathrm{CCl}_{4}$ induced rats after administration of drugs

\begin{tabular}{llll}
\hline Group & $\begin{array}{l}\text { Dose } \\
(\mathbf{m g} / \mathbf{k g})\end{array}$ & $\begin{array}{l}\text { Liver coefficient } \\
(\mathbf{m g} / \mathbf{g})\end{array}$ & $\begin{array}{l}\text { Hyp } \\
(\mu \mathrm{g} / \mathbf{m g} \text { liver })\end{array}$ \\
\hline Normal control & & $53.05 \pm 4.94^{\Delta \Delta \Delta}$ & $0.40 \pm 0.23^{\Delta \Delta}$ \\
$\mathrm{CCl}_{4}$ & & $76.30 \pm 12.64$ & $1.23 \pm 0.49$ \\
Blank-NLC $_{\text {DXMA sol }}$ & 2 & $65.13 \pm 9.5 \mathrm{I}^{\Delta \Delta}$ & $1.34 \pm 1.11$ \\
DXMA sol & $\mathrm{I}$ & $56.07 \pm 4.1 \mathrm{I}^{\Delta \Delta \Delta}$ & $0.43 \pm 0.45^{\Delta \Delta \Delta}$ \\
DXMA-NLC & 2 & $65.25 \pm 9.52^{\Delta \Delta}$ & $0.66 \pm 0.42^{\Delta \Delta \Delta}$ \\
DXMA-NLC & 1 & $60.77 \pm 6.53^{\Delta \Delta \Delta}$ & $0.40 \pm 0.30^{\Delta \Delta \Delta}$ \\
DXMA-NLC & 0.5 & $56.80 \pm 6.90^{\Delta \Delta \Delta, \#}$ & $0.22 \pm 0.39^{\Delta \Delta, \#}$ \\
\hline
\end{tabular}

${ }_{\Delta}^{\Delta}$ significant difference from $\mathrm{CCl}_{4}$ group $(P<0.05)$.

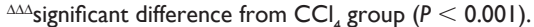

${ }^{*}$ significant difference from DXMA-sol group $(1 \mathrm{mg} / \mathrm{g})(P<0.05)$.

necessary, this hypothesis could explain why DXMA-NLCs generally induced lower liver coefficient and Hyp contents than DXMA.

It should be stressed the dose-effect relationship problem also occurred in our research. As shown in Table 3, among DXMA and DXMA-NLCs groups, the Hyp content $(0.43 \mu \mathrm{g} / \mathrm{mg}$ liver $)$ decreased after injection with a high-dose of DXMA-sol (2 mg $/ \mathrm{kg})$, compared to a low-dose DXMA-sol $(0.66 \mu \mathrm{g} / \mathrm{mg}$ liver, $1 \mathrm{mg} / \mathrm{kg})$, which was consistent with the dose-effect relationship. However, as for DXMA-NLCs, the results were different. The highest anti-inflammatory effects were achieved with the intermediate dose $(1 \mathrm{mg} / \mathrm{kg})$ and low dose of DXMA-NLCs $(0.5 \mathrm{mg} / \mathrm{kg})$. Liver fibrosis is a wound-healing process that is elicited by various toxic stimuli and is always associated with inflammation that contributes to fibrogenesis. ${ }^{28}$ It is also characterized by an excessive deposition of extracellular matrix (ECM) proteins, in which type I collagen predominates. ${ }^{29}$ Hyp is a specific index representing the collagen content of tissues. The inhibition of hydroxyproline levels might reduce the availability of sufficient hydroxyproline required for collagen synthesis. In this study, the lower inhibitory effect caused by the highest dose of DXMA-NLCs $(2 \mathrm{mg} / \mathrm{kg})$ might be explained by the excess concentration of drug. According to the report, the anti-inflammatory effect of glucocorticoids involved in multiple cytokines and mediators of inflammation may result in the complication of the dose-effect relationship. ${ }^{30}$ Some cytokines, such as TNF- $\alpha$, IFN- $\gamma$, and IL- $1 \beta$, could significantly stimulate the ICAM-1 expression in fibroblasts. Dexamethasone (DXM) markedly inhibited these stimulations but the effective concentrations and time were variable. The effect of DXM on TNF- $\alpha$ appeared at the lowest dosage, while for IFN- $\gamma$, the results were highly correlated with the linear 
Table 4 Serum values of AKP, AST, and ALT in $\mathrm{CCl}_{4}$ induced rats after administration of drugs

\begin{tabular}{|c|c|c|c|c|}
\hline Group & Dose (mg/kg) & $\begin{array}{l}\text { AKP } \\
(\mathrm{U} / 100 \mathrm{~mL})\end{array}$ & $\begin{array}{l}\text { AST } \\
(\mathrm{U} / 100 \mathrm{~mL})\end{array}$ & $\begin{array}{l}\text { ALT } \\
(\mathrm{U} / / 00 \mathrm{~mL})\end{array}$ \\
\hline Normal control & & $19.76 \pm 2.27^{\Delta \Delta \Delta}$ & $35.56 \pm 9.42^{\Delta \Delta \Delta}$ & $30.37 \pm 9.19^{\Delta \Delta \Delta}$ \\
\hline $\mathrm{CCl}_{4}$ & & $32.56 \pm 3.02$ & $96.05 \pm 23.12$ & $60.20 \pm 17.65$ \\
\hline Blank-NLC & & $28.74 \pm 4.29$ & $89.94 \pm 16.23$ & $69.37 \pm 21.24$ \\
\hline DXMA sol & 2 & $21.80 \pm 3.04^{\Delta \Delta \Delta}$ & $59.56 \pm 26.49 \Delta \Delta$ & $26.48 \pm 13.06^{\Delta \Delta \Delta}$ \\
\hline DXMA sol & I & $19.51 \pm 3.67^{\Delta \Delta \Delta}$ & $59.20 \pm 12.89^{\Delta \Delta \Delta}$ & $33.21 \pm 6.40^{\Delta \Delta \Delta}$ \\
\hline DXMA NLC & 2 & $19.88 \pm 4.13^{\Delta \Delta \Delta}$ & $62.64 \pm 11.43^{\Delta \Delta}$ & $28.63 \pm 8.0 \mathrm{I}^{\Delta \Delta \Delta}$ \\
\hline DXMA NLC & 1 & $19.62 \pm 3.28^{\Delta \Delta \Delta}$ & $57.90 \pm 25.16^{\Delta \Delta \Delta}$ & $31.09 \pm 11.42^{\Delta \Delta \Delta}$ \\
\hline
\end{tabular}

${ }^{\Delta \triangle}$ significant difference from $\mathrm{CCl}_{4}$ group $(P<0.01)$.

${ }^{\Delta \Delta \Delta}$ significant difference from $\mathrm{CCl}_{4}$ group $(P<0.00 \mathrm{I})$.

dose-effect relationship and for IL-1 $\beta$, the inhibitory effect after administration with DXM varied with time. DXM can increase the stimulant effect of IL-1 $\beta$ at 24 hours but shows an inhibitory effect at 48 hours and there were no significant differences among the different concentration groups. Another report about dose-related effects of dexamethasone on liver damage suggests that high-dose dexamethasone does not provide any additional protective effect on liver damage compared to the low-dose group. ${ }^{31}$ Moreover, high-dose dexamethasone causes obvious growth retardation. Thus, a low dose of corticosteroid provides a significant reduction in liver damage without increased side effects, while a high dose is associated not with weaker fibrosis but with increased side effects. A study of dexamethasone coupled with albumin also demonstrated that at low concentrations $(0.02 \mu \mathrm{M})$, Dexa $_{10}$-HSA was more efficient at inhibiting TNF- $\alpha$ production than uncoupled Dexa. ${ }^{1}$ Therefore, after incorporation into NLCs, DXMA also showed a complicated dosage-effect relationship.

Another interesting thing was that there were no differences for serum levels of AKP, AST, ALT between DXMA-sol and DXMA-NLCs groups, which implies that the inhibiting effect of DXMA-NLCs on hepatic cirrhosis formation may be not directly through protecting liver parenchyma cells. ${ }^{32}$ Further research is being carried out to investigate this.

\section{Conclusion}

Results gained in the present study indicated that DXMA loaded NLCs significantly improved the in vitro release, biodistribution, pharmacokinetic, and therapeutic properties of DXMA. The NLCs remarkably prolonged drug circulation time in plasma and increased drug accumulation in the liver. In research of the anti-inflammatory effects on carrageenan-induced rats and heptoprotective effect on $\mathrm{CCl}_{4}$-induced mice, DXMANLCs exhibited a significant anti-inflammatory and heptoprotective effect compared with DXMA-sol. However, the effect of DXMA-NLCs was not in proportion to the dosage. The intermediate and low dosages presented better anti-inflammatory and heptoprotection effects than DXMA-sol.

\section{Acknowledgments}

The research described above was supported by Ministry of Education (NCET-06-0786), National High-tech R\&D Program (863 Program, 2007AA021801), and National S\&T major project (2009zx09310-002).

\section{Disclosure}

The authors report no conflicts of interest in this work.

\section{References}

1. Barbro NM, Peter O, Vivian KJ, et al. Dexamethasone coupled to albumin is selectively taken up by rat nonparenchymal liver cells and attenuates LPS-induced activation of hepatic cells. J Hepatol. 2000;32:603-611.

2. Sung HK, Dal WC, Choon WK, Sang GK. Lack of therapeutic improvement of liver fibrosis in rats by dexamethasone in spite of ascites amelioration. Chem Biol Interact. 2005;152:37-47.

3. China Pharmacopeial Convention. Chinese Pharmacopoeia. 2005;Section 2:184-185.

4. Zhang Z, Dirk W, Grijpma JF. Poly(trimethylene carbonate) and monomethoxy poly(ethylene glycol)-block-poly(trimethylene carbonate) nanoparticles for the controlled release of dexamethasone. $J$ Control Release. 2006;111:263-270.

5. Carolina GG, Nicolas T, Madeleine B, Amelie B, Elias F. Encapsulation of dexamethasone into biodegradable polymeric nanoparticles. Int J Pharm. 2007;331(2):153-159.

6. Gomez-Gaete C, Fattal E, Silva L, Besnard M, Tsapis N. Dexamethasone acetate encapsulation into Trojan particles. J Control Release. 2008;128(1):41-49.

7. Joshi MD, Müller RH. Lipid nanoparticles for patrnteral delivery of actives. Eur J Pharm Biopharm. 2009;71:161-172.

8. Sun JY, Zhou ZF, Liu F, Chen GS. Pharmacokinetics and tissue distribution of oxymatrine-SLN. Chin Pharm J. 2007;42:1091-1095.

9. Yu BT, Zhang ZR, Zeng RJ. Study on the liver targeted 5-fluorouracil solid lipid nanoparticles. Yао Хие Хие Вао. 2000;35:704-705. 
10. Lu B, Xionga SB, Yanga H, Yina XD, Chao RB. Solid lipid nanoparticles of mitoxantrone for local injection against breast cancer and its lymph node metastases. Eur J Pharm. Sci. 2006;28:86-95.

11. Lange M, Malyusz M. Improved determination of small amounts of free hydroxyproline in biological fluids. Clin Chem. 1994;409:1735-1738.

12. $\mathrm{Mu} \mathrm{L}$, Feng SS. Fabrication, characterization and in vitro release of paclitaxel (Taxol) loaded poly (lactic-co-glycolic acid) microspheres prepared by spray drying technique with lipid/cholesterol emulsifiers. J Control Release. 2001;76:239-254.

13. Lu W, He LC, Wang CH, Li YH, Zhang SQ. The use of solid lipid nanoparticles to target a lipophilic molecule to the liver after intravenous administration to mice. Int J Biol Macromol. 2008;43:320-324.

14. Scholer N, Olbrich C, Tabatt K, Müller RH, Hahn H, Liesenfeld O. Surfactant, but not the size of solid lipid nanoparticles (SNLC) influences viability and cytokine production of macrophages. Int J Pharm. 2001;221:57-67.

15. Scholer N, Hahn H, Müller RH, Liesenfeld O. Effect of lipid matrix and size of solid lipid nanoparticles (SNLC) on the viability and cytokine production of macrophages. Int J Pharm. 2002;231:167-176.

16. Reddy LH, Sharma RK, Chuttani K, Mishra AK, Murthy RSR. Etoposide incorporated tripalmitin nanoparticles with different surface charge: formulation, characterization, radiolabeling, and biodistribution studies. AAPS J. 2004;6.

17. Fundarò A, Cavalli R, Bargoni A, Vighetto D, Zara GP, Gasco MR. Nonstealth and stealth solid lipid nanoparticles (SLN) carrying doxorubicin: pharmacokinetics and tissue distribution after i.v. administration to rats. Pharmacol Res. 2000;42:337-343.

18. Gert S, Sheila OB, Toos D, Danilo DL. Surface modification of nanoparticles to oppose uptake by the mononuclear phagocyte system. Adv. Drug Deliv Rev. 1995;17:31-48.

19. Müller RH, Maaseen S, Schwa C, Mehnert W. Solid lipid nanoparticles (SLN) as potential carrier for human use: interaction with human granulocytes. J Control Release. 1997;47:261-269.

20. Junzo S, Satoru S, Akira S, Hiroshi F, Hideki S, Tadanori M. A nanometer lipid emulsion, lipid nano-sphere $\left(\mathrm{LNS}^{\circledR}\right)$, as a parenteral drug carrier for passive drug targeting. Int J Pharm. 2004;273:75-83.
21. Rudt S, Müller RH. In vitro phagocytosis assay of nano- and microparticles by chemiluminescence.I. Effect of analytical parameters, particle size and particle concentration. J Control Release. 1992;22:263-272.

22. Rudt S, Müller RH. In vitro phagocytosis assay of nano- and microparticles by chemiluminescence.II. Effect of surface modification by coating of particles with poloxamer on the phagocytic uptake. J Control Release. 1993a;25:51-59.

23. Rudt S, Müller RH. In vitro phagocytosis assay of nano- and microparticles by chemiluminescence. III. Uptake of differently sized surface-modified particles, and itscorrelation to particle properties and in vivo distribution. Eur J Pharm Sci. 1993b;1:31-39.

24. Li JC, Sha XY, Zhang LJ, Fang XL. 9-Nitrocamptothecin nanostructured lipid carrier system: in vitro releasing characteristics, uptake by cells, and tissue distribution in vivo. Yао Хие Хие Вао. 2005;40:970-975.

25. Esposito M, Fantin MM, Drechsler L, et al. Solid lipid nanoparticles as delivery systems for bromocriptine. Pharm Res. 2008;25:1521-1530.

26. Quan DQ, Cui GH, Dong HJ, Ruan JX. Tissue distribution and antiinflammatory activity of dexamethansone acetate incorporated in lipid emulsion. J Chin Pharm Sci. 2001;10:90-93.

27. Lin HM, Tseng HC, Wang CJ, Lin JJ, Lo CW, Chou FP. Hepatoprotective effects of Solanum nigrum Linn extract against CC14-iduced oxidative damage in rats. Chem Biol Interact. 2008;171:283-293.

28. Park O, Jeong W, Wang L, et al. Diverse roles of invariant natural killer T cells in liver injury an fibrosis induced by carbon tetrachloride. Hepatology. 2009;49:1683-1694.

29. Friedman SL. Molecular regulation of hepatic fibrosis, an integrated cellular response to tissue injury. J Biol Chem. 2000;2754:2247-2250.

30. Huang Q, Zou DJ, Guo ZR. Effects of dexamethasone on the cultured retroocular fibroblast stimulated by cytokines. Chin J Endocrinol Metab. 2000;16:224-227.

31. Halil E, Hayrettin O, Hulya O, Huseyin B. Dose-related effects of dexamethasone on liver damage due to bile duct ligation in rats. World $J$ Gastroenterol. 2006;12:5379-5383.

32. MuY, Liu P, Du G, et al. Action mechanism of Yi Guan Jian Decoction on $\mathrm{CCl}_{4}$ induced cirrhosis in rats. J Ethnopharmacol. 2009; 121:35-42.
International Journal of Nanomedicine

\section{Publish your work in this journal}

The International Journal of Nanomedicine is an international, peerreviewed journal focusing on the application of nanotechnology in diagnostics, therapeutics, and drug delivery systems throughout the biomedical field. This journal is indexed on PubMed Central,

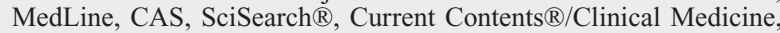

\section{Dovepress}

Journal Citation Reports/Science Edition, EMBase, Scopus and the Elsevier Bibliographic databases. The manuscript management system is completely online and includes a very quick and fair peer-review system, which is all easy to use. Visit http://www.dovepress.com/ testimonials.php to read real quotes from published authors. 